\title{
EXPERIMENTAL ROBUST CONTROL STUDIES ON AN UNSTABLE MAGNETIC SUSPENSION SYSTEM
}

\author{
Kyong B. Lim* and David E. Cox ${ }^{\dagger}$ \\ NASA Langley Research Center \\ Hampton, Virginia
}

\section{Summary}

This study is an experimental investigation of the robustness of various controllers designed for the Large Angle Magnetic Suspension Test Fixture (LAMSTF). Both analytical and identified nominal models are used for designing controllers along with two different types of uncertainty models. Robustness refers to maintaining tracking performance under analytical model errors and dynamically induced eddy currents, while external disturbances are not considered. Results show that incorporating robustness into analytical models gives significantly better results. However, incorporating incorrect uncertainty models may lead to poorer performance than not designing for robustness at all. Designing controllers based on accurate identified models gave the best performance. In fact, incorporating a significant level of robustness into an accurate nominal model resulted in reduced performance. This paper discusses an assortment of experimental results in a consistent manner using robust control theory.

\section{Introduction}

Robust tracking control of the Large Angle Magnetic Suspension Test Fixture (LAMSTF) consists of controlling the attitude and position of a suspended rigid body in the presence of external disturbances and model uncertainties. A detailed description of the LAMSTF is given in [1] and the open-loop dynamic properties of the magnetic suspension system is given in [2].

The work reported herein parallels references [3][6] and is a continuation of [7]. The above references consider vibration attenuation and fine-pointing control for a stable large flexible laboratory structure. In stark contrast to the above passively stable flexible structure, the LAMSTF system is a highly unstable rigid body. Furthermore, the nature of the uncertainties in the two systems differ; the uncertainty in the system in [3][6] is mostly due to inaccurate knowledge of damping, frequency and mode shapes of the structural modes and truncated higher frequency structural modes. While for LAMSTF the uncertainty is mostly due to errors in the linearization about the equilibrium state, an inaccurate knowledge of the spatial distribution of the magnetic field, sensor calibration errors, and errors at the plant input due to induced eddy currents.

The underlying motivation in this study is that

\footnotetext{
*Research Engineer, SCB, FDCD, cox@scb1.larc.nasa.goy

${ }^{\dagger}$ Research Engineer, SCB, FDCD, lim@scb6.larc.nasa.gov
}

for a class of complex expensive systems, for example space vehicles, there is a limitation on model fidelity due to time-varying model variations and/or limited amount of data available for sufficiently thorough system identification work. The novelty in this paper consists of determining what factors are important in applying robust control design to a real problem.

A series of tests was designed and conducted to investigate the effect of erroneous nominal models and erroneous uncertainty models. The effect of erroneous nominal model was investigated by comparing the closed loop results of controllers based on analytical and identified plants. The effect of erroneous uncertainty model was investigated by comparing the closed loop results of nominal to a perturbed test which involves placing an aluminium ring above the suspended magent to induce a particular type of eddy current. This externally induced eddy current is in addition to the existing eddy currents in the five iron cores of the electromagents which is not included in the analytical model.

\section{Linear Model of LAMSTF}

The system shown in Figure 1 is the LAMSTF. It consists of five electromagnets which actively suspend a small cylindrical permanent magnet. The cylinder is a rigid body and has six independent degrees of freedom. Reference [7] gives a detailed derivation of both the linear

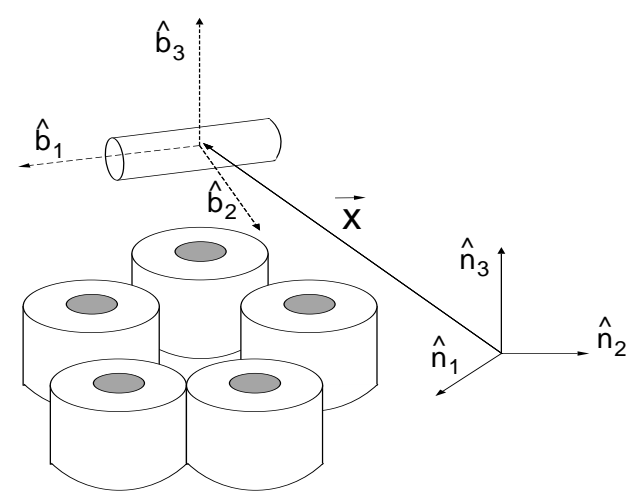

Figure 1: LAMSTF Configuration

and nonlinear models. The following provides a synopsis for the linear model used in the control design. 
Defining the state vector

$$
\xi=\left(\omega_{2}, \omega_{3}, \beta_{2}, \beta_{3}, v_{1}, v_{2}, v_{3}, x_{1}, x_{2}, x_{3}\right)^{T}
$$

the linearized perturbed motion about the equilibrium is given by

$$
\delta \dot{\xi}=\hat{A} \delta \xi+\hat{B} \delta \rho
$$

where the detailed analytical expressions for $\hat{A}$ and $\hat{B}$ are given in [7]. These expressions depend on many physical constants including an analytic approximation of the magnetic field distribution whose numerous parameters were obtained by numerical modeling. The variables $\omega_{i}$, $\beta_{i}, v_{i}$, and $x_{i}$ denote the $i$ th angular velocity of the cylinder with respect to the body frame; the ith Euler parameter relative to the inertial frame; and the $i$ th translational velocity and displacement of the centroid, respectively.

It can be shown that all ten states are completely controllable from the five coil currents. The open-loop system at the equilibrium state is also very unstable. For a detailed discussion of the physical significance of all modes, the interested reader is referred to [2].

A set of six sensors detects the five physical variables: pitch and yaw angles, and three displacements of the centroid. Roll of the cylinder is both uncontrollable and unobservable. The five physical variables sensed, denoted as $y^{\prime}$, are related approximately linearly to the state vector by

$$
y^{\prime}=\hat{C} \delta \xi
$$

where $\hat{C}$ is given in [7]. The measured voltage outputs denoted by $y$, are related to physical states $y^{\prime}$ by

$$
y^{\prime}=[\mathrm{S} 2 \mathrm{P}] y \quad y=[\mathrm{P} 2 \mathrm{~S}] y^{\prime}
$$

Both the sensor (physical state to output voltage) and actuator systems (current to forces and torques) have high bandwidth and are modeled as constants (not shown for brevity), denoted by matrices $P 2 S$ and $A 2 F$. In summary, input to the system consists of five currents into five electromagnets, $\delta \rho$, and the measured outputs are six voltage signals, $y$, related to the position of the suspended element.

\section{Controller Design}

The main objective of the controller is to stabilize the cylinder and track a command signal about the equilibrium state. The problem is complicated by the omni presence of model errors and noise. Hence, robust tracking is sought. This requires the specification of a tracking performance index and the uncertainty set for which the tracking performance is supposedly guaranteed. Of course, this guarantee is with respect to modeled uncertainty which itself may be uncertain. Uncertainty in the uncertainty model itself is a real dilemma which partly motivates our work.

Controllers that meet a certain tracking performance are sought for a set of plants. The set of plants is defined by the nominal and uncertainty models. Both the linearized analytical model derived from first principles and an empirical model obtained from closed loop system identification [8] results are used as the nominal models. The measurement noise and disturbance uncertainties are included to reflect realistic values from signals encountered in the laboratory. On the other hand, model uncertainties in the loop are more difficult to characterize quantitatively. The model errors considered consist of two components, namely, nominal model error and externally induced eddy currents (due to added aluminium ring). No attempt was made to model the deviation of the nominal model from the true model but instead controllers based on identified and analytical nominal models are compared. On the other hand, errors due to externally induced eddy currents are heuristically modeled resulting in two different types of uncertainties, multiplicative at the input and additive.

Figure 2 illustrates the interconnection structure. Due to the high speed of the real-time digital implemen-

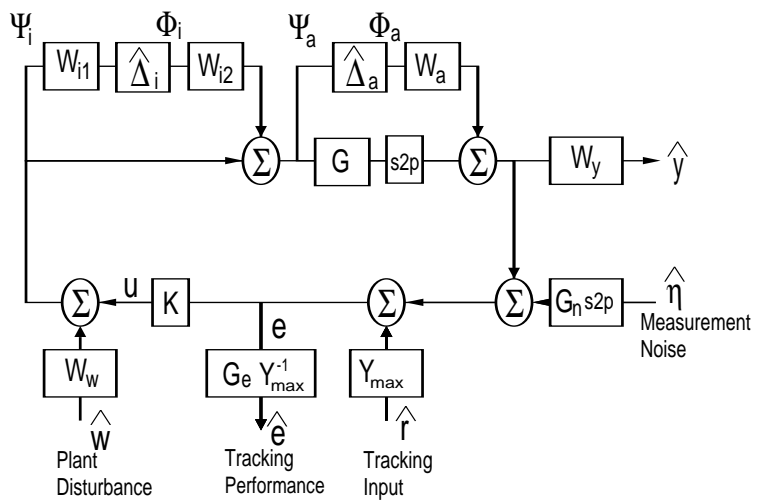

Figure 2: Interconnections in LAMSTF system

tation at $800 \mathrm{~Hz}$ when compared to the loop bandwidth of approximately $50 \mathrm{~Hz}$, all controllers were designed in the continuous domain. The small computational time delay and zero-order-hold effects are ignored.

\subsection{Tracking Performance}

The tracking performance is defined by the principal gains such that

$$
\bar{\sigma}\left[G_{e}^{-1} Y_{\max }^{-1}(I+G K)^{-1} Y_{\max }\right] \leq 1 ; \forall \omega \in[0, \infty)
$$

where the frequency weighting function matrix, $G_{e}=$ $\operatorname{diag}\left(g_{e 1}, \ldots, g_{e 5}\right)$, are given by

$$
g_{e}=f_{D C}\left[\frac{\left(s+\omega_{n}\right)}{\left(s+\omega_{d}\right)}\left(\frac{\omega_{d}}{\omega_{n}}\right)\right]
$$

The following parameters were chosen: $\omega_{n}=2 \mathrm{r} / \mathrm{s}$ for pitch and yaw axes and $1 \mathrm{r} / \mathrm{s}$ for $x, y, z$ axes, $\omega_{d}=1000$ $\mathrm{r} / \mathrm{s}$ and $f_{D C}=.05$ for all channels. This weight specifies the steady state tracking error to be within $5 \%$ for all five axes up to about 1 to $2 \mathrm{rad} / \mathrm{sec}$ and decreases by 20 $\mathrm{db}$ per decade until $1000 \mathrm{rad} / \mathrm{s}$. This frequency weighting results in the tracking error reaching $100 \%$ at a frequency of about $200 \mathrm{rad} / \mathrm{s}$ for both controllers.

Note that the above sensitivity matrix is normalized to unity at the inputs and outputs by the designed tracking command magnitudes

$$
Y_{\text {max }}=\operatorname{diag}\left(\frac{\pi}{180}, \frac{\pi}{180}, .0005, .0005, .0005\right)
$$

Therefore the maximum singular value corresponding to this scaled transfer function from command input to tracking error can be interpreted as the maximum 2-norm error with respect to all unit 2-norm bounded tracking command vectors, scaled by $Y_{\max }$. 


\begin{tabular}{|l||l|}
\hline Analytical Model & Identified Model \\
\hline$-0.0000-0.9556 \mathrm{i}$ & 4.0290 \\
$-0.0000+0.9556 \mathrm{i}$ & $-3.1592-5.3601 \mathrm{i}$ \\
$0.0000-7.9697 \mathrm{i}$ & $-3.1592+5.3601 \mathrm{i}$ \\
$0.0000+7.9697 \mathrm{i}$ & -7.6822 \\
-9.7764 & $-0.8034-10.2975 \mathrm{i}$ \\
9.7764 & $-0.8034+10.2975 \mathrm{i}$ \\
57.8061 & $-58.8031-0.4229 \mathrm{i}$ \\
-57.8061 & $-58.8031+0.4229 \mathrm{i}$ \\
58.7793 & 60.3627 \\
-58.7793 & 62.3473 \\
\hline
\end{tabular}

Table 1: Eigenvalues for analytic and identified models

\subsection{Uncertainty Models}

In this study, the main source of model errors are due to (1) a relatively inaccurate analytical model which is used as a nominal model and (2) externally induced eddy current effects due to the placement of an aluminum ring about the $\mathrm{z}$ axis.

To investigate the first effect, an identified model of the plant without the externally induced eddy currents was obtained by a closed loop system identification technique. Table 1 shows the poles of the analytical and identified models. Neither model contains transmission zeros. A significant difference between the analytical and identified model is noted, especially at lower frequencies. Instead of trying to generate an uncertainty model from these two models, separate controllers were designed using different nominal models.

To investigate the second effect, two types of uncertainties were developed and tested. Only the multiplicative uncertainty description was designed to have some physical basis for the actual eddy current effects. In this way, the effect of using an incorrect uncertainty model can be experimentally evaluated.

\subsubsection{Additive}

The additive uncertainty model (Figure 2) maps current inputs to physical outputs. Assuming a nominal current of $1 \mathrm{amp}$ input in all channels, the perturbed currents are scaled by the maximum anticipated physical outputs and multiplied by a small factor, $f_{a}=.003$ and .00015 , for significant and insignificant additive uncertainty cases. While the uncertainty in all other axes are assumed constants, the additive uncertainty in the pitch axis is rolled up by a first order polynomial of the form $p_{a}=$ $(s+1) /(s+100 \pi) 100 \pi$ to simulate the effect of eddy currents on the pitch axis. The additive uncertainty weight is chosen as

$$
W_{a}=f_{a} * \operatorname{diag}\left(p_{a}, 1,1,1,1\right)
$$

\subsubsection{Multiplicative at Input}

Figure 3 shows the eddy current uncertainty modeled as a multiplicative type at the input. It is known that eddy currents are proportional to the rate of change of the magnetic field and therefore rate of change of actuator currents. This eddy current in turn produces its own magnetic field which interacts with the suspended magnet to produce perturbation forces. These perturbed forces

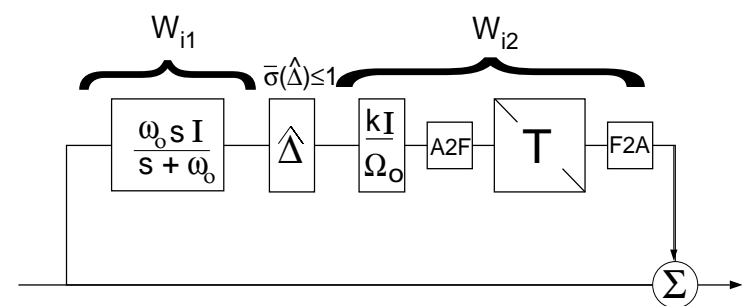

Figure 3: Input Multiplicative Uncertainty

and moments can be modeled as a set of perturbations in the actuator currents. By knowing that the induced eddy currents affect mostly the pitch axis, the moments associated with the pitch axis were weighted 40 times more than the other axes, i.e., $T=\operatorname{diag}(4, .1, .1, .1, .1)$. It is important to note that the 40 times weighting used is still arbitrary in the sense that it is simply our representation of relative dominance. This is an example of an uncertainty dilemma in modeling uncertainties. The 5 by 5 unstructured uncertainty represents the unknown complex relationship between the time rate of change in the five electromagnet currents to the equivalent perturbed electromagnet currents.

The pure differentiator is approximated by a proper transfer function $\omega_{0} s /\left(s+\omega_{0}\right) I_{5 \times 5}$ where $\omega_{0}=1000$ $\mathrm{rad} / \mathrm{sec}$. The parameters $k / \Omega_{0}$ denote the expected amplitude of the eddy currents, and the values $k=.2$ and .01 were chosen to represent significant and insignificant eddy current cases. The parameter $\Omega_{0}=100 \pi \mathrm{rad} / \mathrm{sec}$ denotes the reference frequency with respect to which the magnitude of $k$ is scaled. The constant matrices $[A 2 F]$ and $[F 2 A]$ (not shown for brevity) denote the transformation from coil current to torques and forces on the rigid body and vice versa.

\subsubsection{Input Disturbance and Noise}

The input disturbances to the plant are included to account for bias currents and amplifier noise, a maximum amplitude of $.1 \mathrm{amp}$ is assumed in all channels over all frequencies. To normalize the input disturbance to a unit 2-norm, the diagonal weighting matrix is given by $W_{w}=1 / \sqrt{5} I_{5 \times 5}$.

Although the sensing system provides a wide dynamic range and is modeled as a constant matrix, $[S 2 P]$ (not shown for brevity), there are uncertainties due to measurement noise, small angle/displacement assumptions, sensor offsets from equilibrium, and calibration errors. In addition, the closed-loop bandwidth can be limited by penalizing high frequency control activity via assuming a fictitious large noise at high frequencies. The measurement noise weighting matrix chosen consists of $W_{n}=G_{n} *[S 2 P]$ where the first term transforms assumed measurement noise voltages into physical displacements while $G_{n}=g_{n} * I_{6 \times 6}$ where

$$
g_{n}=\left(\frac{s+100}{s+5000}\right) * 50 * N_{s d}
$$

The term $N_{s d}=0.04$ denotes 2-sigma noise level in the voltage measurements observed in the laboratory. The output is normalized by choosing $W_{y}=Y_{\max }^{-1}$.

\subsection{Control Designs Implemented}

Figure 4 shows the augmented plant which includes the performance and uncertainty weights. The $\mu$ was 


\begin{tabular}{|c|c|c|}
\hline $5 \times 1$ & \multirow{3}{*}{$\begin{array}{l}\text { Augmented } \\
\text { Plant }\end{array}$} & $5 \times 1$ \\
\hline $5 \times 1$ & & $5 \times 1$ \\
\hline & & $6 \times 1$ \\
\hline $5 \times 1$ & & $5 \times 1$ \\
\hline $5 \times 1$ & & $5 \times 1$ \\
\hline
\end{tabular}

Figure 4: Inputs and outputs of the augmented plant

minimized using D-K iteration [9, 10, 11]. All designs converged in less than 5 iterations. The MATLAB toolbox, $\mu$-Tools [12] was used.

Six controllers, $K_{a a p}, K_{a a r}, K_{a m p}, K_{a m r}, K_{i m p}$, $K_{i m r}$, are designed and tested with and without the externally induced eddy currents, which gives a total of twelve experiments. The three subscripts for the controllers are defined as follows: the first subscript $(a, i)$ denotes the analytic and identified models, respectively, for the nominal plant; the second subscript $(m, a)$ denotes the multiplicative and additive uncertainties; and the third subscript $(p, r)$ denotes the performance and robustness weightings, respectively. More precisely, the performance weighted controller (third subscript $p$ ) is actually a controller with negligible built-in robustness while the robustness weighted controller (third subscript $r$ ) is designed with a significant level (compared to performance weighted case) of uncertainties assumed.

The order of the controllers was reduced to ease real-time implementation and to remove states that are very weakly controllable and observable. The order of the controllers was reduced to approximately 30 states from 40 or more states. In all cases, the reduced controller did not significantly degrade the predicted robust performance.

In the design of controllers, since only the assumed level of uncertainties differ, the robust performance index, $\mu$, for performance controllers produced significantly smaller values than robustness emphasized controllers. All suboptimal $\mu$ values for the performance controllers easily satisfied the constraints while the suboptimal $\mu$ values for the robustness emphasized controllers did not satisfy the desired level of robust performance up to a factor of 2.6 for a robust controller with additive uncertainties $\left(K_{a a r}\right)$. However, since $\mu$ is defined relative to different incorrect nominal models and incorrect uncertainty structures, comparing the relative $\mu$ values attained do not necessarily translate to performance for the unknown real system. The identified model provides an accurate nominal model but the precise level and the uncertainty structure of the externally induced eddy currents are still unknown.

\section{Results}

Figure 6 shows the results from a typical tracking experiment which consists of independent step commands in all five axes. The step commands were $2^{\circ}$ for pitch and yaw and $2 \mathrm{~mm}$ for $\mathrm{x}, \mathrm{y}$, and $\mathrm{z}$ axes. Although there is considerable overshoot in all axes, the damping and steady state errors were satisfactory.

Since the aluminum ring predominantly affects the pitch axis, only pitch responses are shown in Figures 7 9. The simulated, nominal experiment (without added ring) and perturbed experiment (with ring) for controllers
$K_{\text {aap }}$ and $K_{\text {aar }}$ are given in Figure 7 and similarly for controllers $K_{a m p}$ and $K_{a m r}$ in Figure 8 and controllers $K_{i m p}$ and $K_{i m r}$ in Figure 9.

The simulations shown do not include the external source of eddy currents induced by the added aluminum ring so that it is only compared to the nominal experiments. Comparing performance controllers in Figures 7 to 9 shows that the simulated response for the identified model (Figure 9) is much closer to the corresponding experiment than the analytical models (Figures 7 and 8). It therefore appears that the identified model is closer to the true nominal system than the analytical model.

\subsection{Errors in Uncertainty Model}

Controllers $K_{a a p}$ and $K_{a a r}$ in Figure 7 assume additive uncertainties while controllers $K_{a m p}$ and $K_{a m r}$ in Figure 8 assume a multiplicative form. However, only the "robust" controllers, $K_{a a r}$ and $K_{a m r}$ incorporate significant uncertainty levels. These figures show that including multiplicative uncertainty enhances performance (cf $K_{a m r}$ vs. $K_{a m p}$ in Figure 8 for perturbed experiments) while including additive uncertainty degrades performance ( $\mathrm{cf}$ $K_{\text {aar }}$ vs. $K_{\text {aap }}$ in Figure 7 for perturbed experiments). Clearly, the choice of an uncertainty model is very significant and it is the burden of the control designer to employ physical insight when applying robust control techniques.

\subsection{Errors in Nominal Model}

The nominal experiments for controllers $K_{i m p}$ and $K_{a m p}$ (Figures 8 and 9) show that controllers based on more accurate nominal models give significantly better performance in terms of overshoot and damping. However for robust controllers, $K_{i m r}$ and $K_{a m r}$, the controller based on a more accurate nominal model gave only slightly better performance. This effect is amplified for the perturbed experiments which can be thought of as larger errors in both the nominal models.

\subsection{Performance and Robustness Tradeoff}

Figure 5 depicts the anticipated performance variations due to differences between the nominal $\left(G_{0}\right)$ and true models $\left(G_{T}\right)$ for differing design uncertainties $(\Delta)$. The predicted performance drops with the distance of the true model from the nominal model. The robustness tradeoff involves a reduction in overall performance in order to guarantee reduced levels of performance for a larger set of plants $\left(\Delta_{r}\right)$.

All the experimental results shown in this paper can be explained by the generic performance and robustness tradeoff diagram shown in Figure 5. Three main observations are:

- Let $G_{0}$ be the analytic model and $G_{2}=G_{T}$. Since $\left\|\Delta_{p}\right\|_{\infty} \leq\left\|G_{2}-G_{0}\right\|_{\infty} \leq\left\|\Delta_{r}\right\|_{\infty}, K_{a m p}$ could give poor performance while $K_{\text {amr }}$ should maintain a reduced (by tradeoff) level of performance. In addition, $K_{a m p}$ is expected to be more sensitive (higher slope in Figure 5) to eddy current effects than $K_{a m r}$. These predictions are all verified in Figure 8.

- Let $G_{0}$ be the identified model and $G_{1}=G_{T}$. Since $\left\|G_{1}-G_{0}\right\|_{\infty} \leq\left\|\Delta_{p}\right\|_{\infty}, K_{i m r}$ should give less performance than $\bar{K}_{i m p}$. With eddy currents, there is only a slight reduction in performance (shaded region around $G_{1}$ in Figure 5). Figure 9 is consistent 


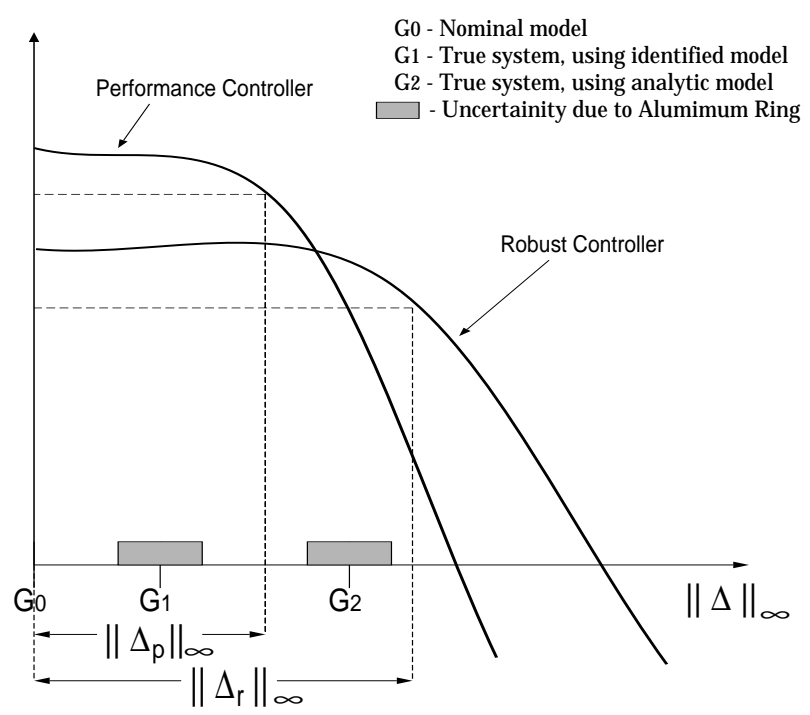

Figure 5: Effect of model uncertainty on robustness/performance tradeoff

with the above explanation. Clearly, in this case a performance tradeoff for robustness is unnecessary.

- Controller $K_{a m r}\left(G_{2}=G_{T}\right)$ should have similar performance as $K_{i m r}\left(G_{1}=G_{T}\right)$ since both are robust controllers and they satisfy $\left\|G_{0}-G_{T}\right\|_{\infty} \leq$ $\left\|\Delta_{r}\right\|_{\infty}$. This is reasonably verified by comparing Figures 8 and 9 . Note that for $K_{a m r}$, the true plant may lie on the shoulder (Figure 5) and is slightly more sensitive to eddy currents than controller $K_{i m r}$.

\section{Concluding Remarks}

Although nothing concrete has been proven, the results of this study provide a better understanding of the major factors that influence the degree of success of a robust control approach when applied to real problems. In particular, the experiments were tailored to study the effects of erroneous nominal and uncertainty models. The experimental results support the following hypothesis: (1) Incorporating robustness into analytical models gives significantly better results. However, incorporating incorrect uncertainty models can lead to poorer performance than not designing for robustness at all. (2) Designing controllers based on accurate identified models gives good performance without robustness considerations. Finally it was found that the performance obtained via robust control techniques using only an inaccurate analytical model with an approximate uncertainty model is comparable to the controller performance obtained by using an accurately identified model but without robustness considerations.

\section{References}

1. Groom, N.J., and Britcher, C.P., "A Description of a Laboratory Model Magnetic Suspension Test Fixture with Large Angular Capability," First IEEE Conf. on Control Applications, Dayton, Ohio, 1992.

2. Groom, N.J., and Britcher, C.P., "Open-Loop Characteristics of Magnetic Suspension Systems Using Electro-
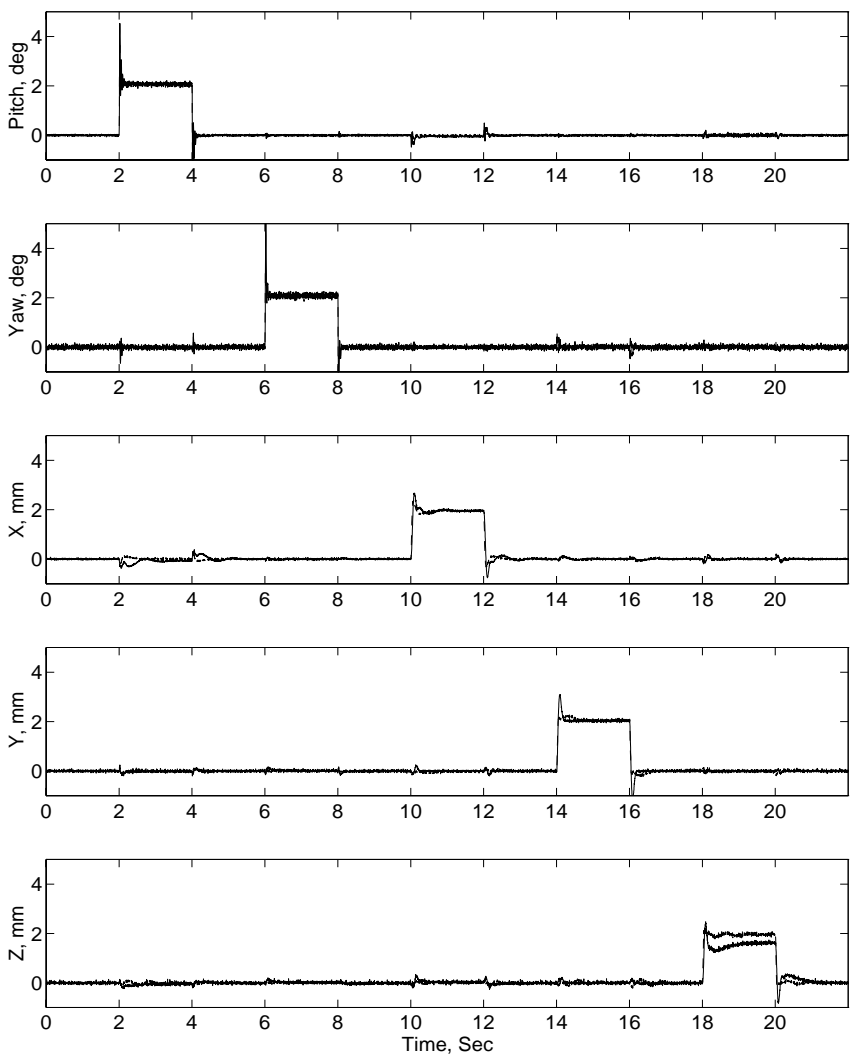

Figure 6: Tracking response for $K_{a m r}$ and $K_{i m p}$

magnets Mounted in a Planar Array," NASA-TP 3229, November 1992.

3. Balas, G.J., and Doyle, J.C., "Robust Control of Flexible Modes in the Controller Crossover Region," ACC, 1989.

4. Balas, G.J., and Doyle, J.C., "Robustness and Performance Tradeoffs in Control Design for Flexible Structures," $C D C, 1990$.

5. Lim, K.B., Maghami, P.G., and Joshi, S.M., "A Comparison of Controller Designs for an Experimental Flexible Structure," IEEE Control System Magazine, Vol.12, No.3, June 1992.

6. Lim, K.B., and Balas, G.J., "Line-of-Sight Control of the CSI Evolutionary Model: $\mu$ Control," ACC, 1992.

7. Lim, K.B., and Cox, D.E., "Robust Tracking Control of a Magnetically Suspended Rigid Body," 2nd Int. Symp. on Magnetic Suspension Technology, 1993.

8. Huang,J-K., M-H Hsiao, M-H., and Cox, D.E., "Identification of Linear Stochastic Systems from Closed-Loop Data with Known Feedback Dynamics," CDC, 1994.

9. Doyle, J.C., "Analysis of Feedback Systems with Structured Uncertainties," Proc. IEE-D 129, 1982, pp. 242250 .

10. Doyle, J.C., Glover, K., Khargonekar, P., and Francis, B., "State-space Solutions to Standard $\mathrm{H}_{2}$ and $\mathrm{H}_{\infty}$ Control Problems," IEEE Trans on Auto Contr, Vol.34, No.8, August 1989.

11. MUSYN Robust Control Short Course Lecture Notes, Arcadia, CA, September, 1989.

12. Balas, G.J., Doyle, J. D., Glover, K., Packard, A. K., and Smith, R., $\mu$-Analysis and Synthesis Toolbox, MUSYN Inc., Minneapolis, 1991. 

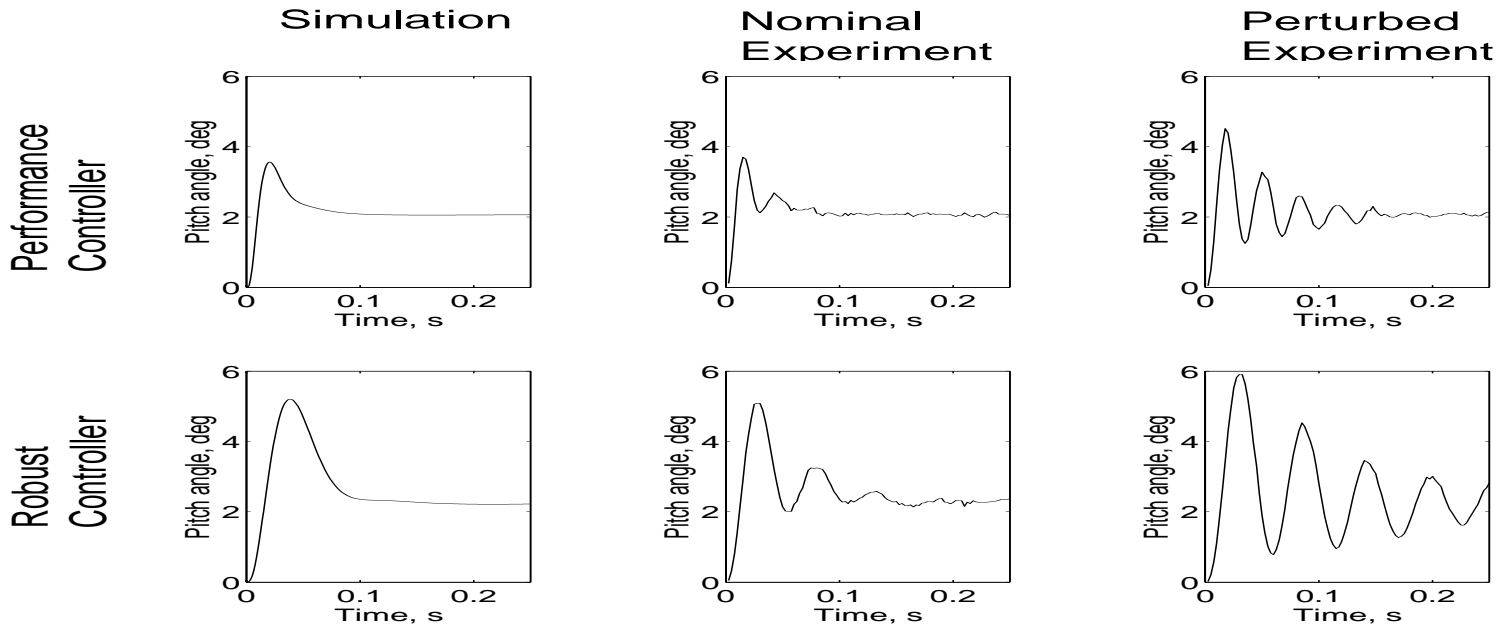

Figure 7: Experimental and simulated pitch tracking for $K_{a a p}$ and $K_{a a r}$
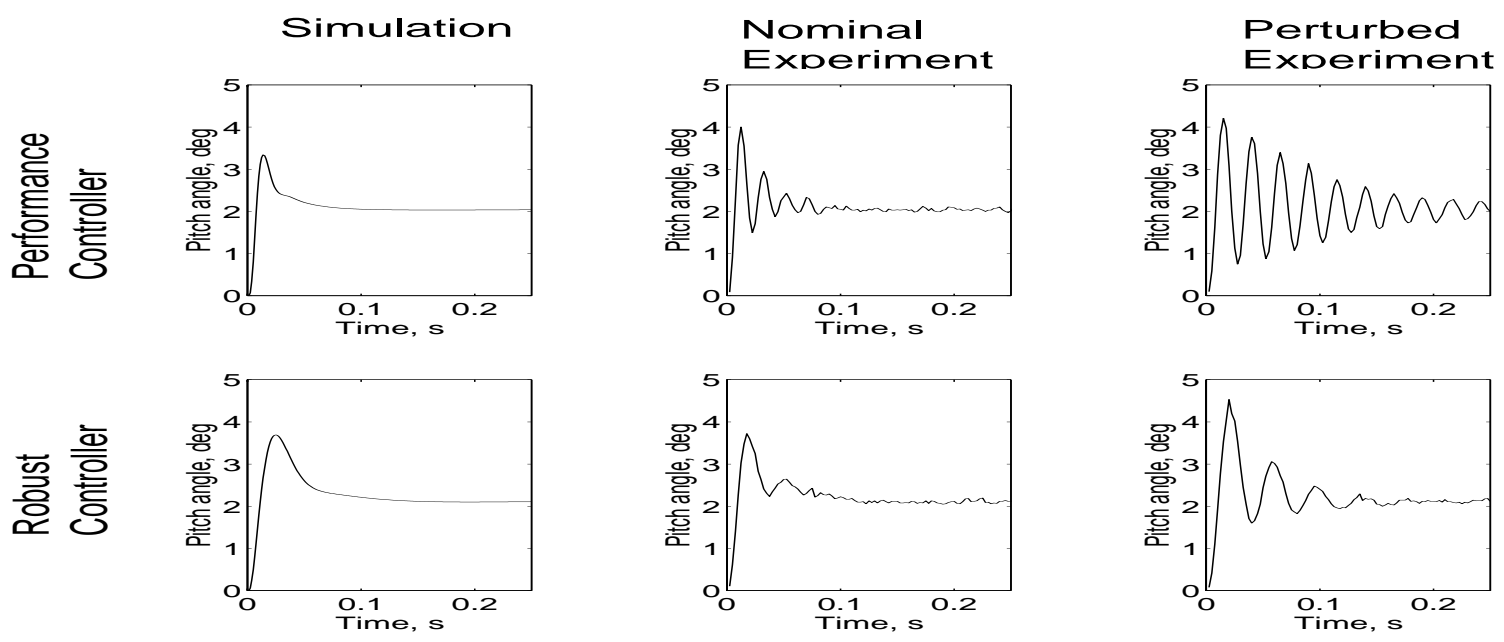

Figure 8: Experimental and simulated pitch tracking for $K_{a m p}$ and $K_{a m r}$
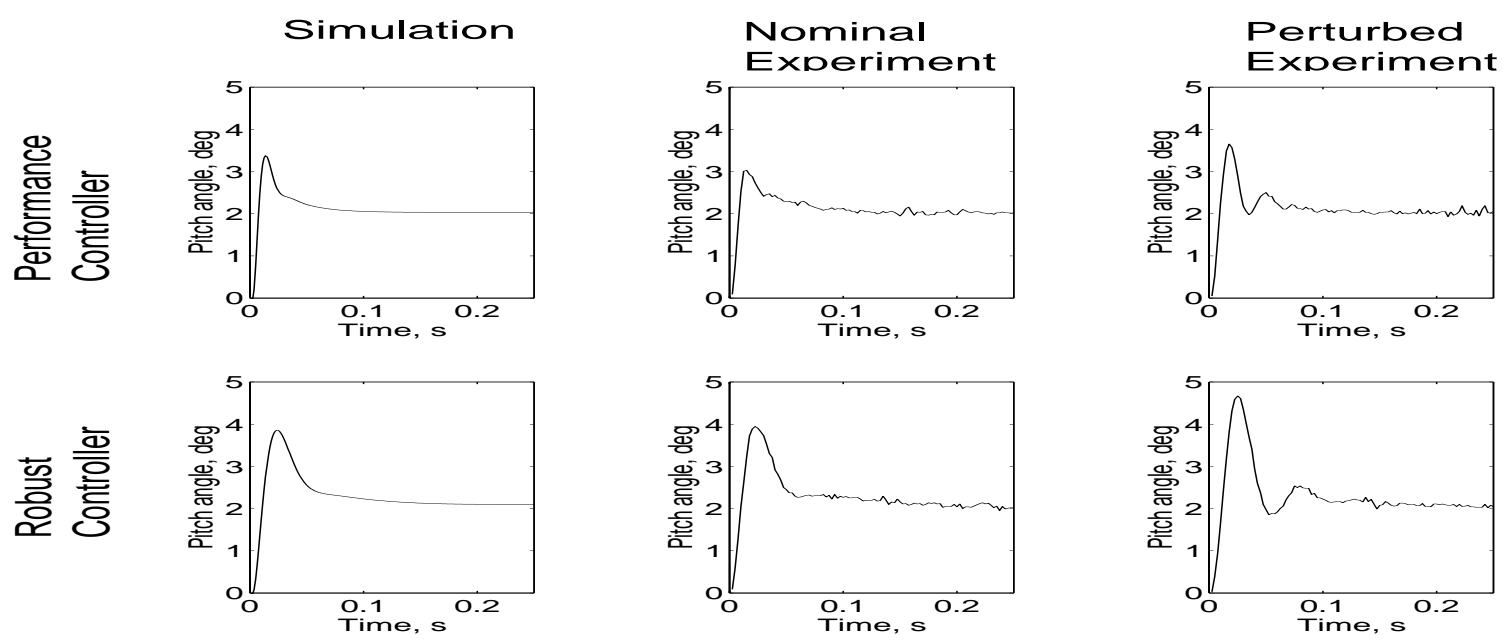

Figure 9: Experimental and simulated pitch tracking for $K_{i m p}$ and $K_{i m r}$ 